\title{
Flexor Digitorum Superficialis Muscle
}

National Cancer Institute

\section{Source}

National Cancer Institute. Flexor Digitorum Superficialis Muscle. NCI Thesaurus. Code C150850.

A muscle that originates in the humeroulnar and radial heads of the forearm and inserts in the middle phalanges of the second through fifth digits of the hand; it is primarily involved in the flexion of the fingers at the proximal interphalangeal joints and is supplied by the median nerve and the ulnar artery. 